Review

\title{
Recent Progress in Characterizing Long Noncoding RNAs in Cancer Drug Resistance
}

\author{
Wenyuan Zhao1, Bin Shan², Dan He³, Yuanda Cheng4, Bin Li1 ${ }^{1}$, Chunfang Zhang4, Chaojun Duan ${ }^{1,5,6 \bowtie}$ \\ 1. Department of Oncology, Xiangya Hospital, Central South University, Changsha, PR China; \\ 2. College of Medical Sciences, Washington State University Spokane, WA, USA; \\ 3. Hunan Cancer Hospital, The Affiliated Tumor Hospital of Xiangya Medical College, Central South University, Changsha, PR China; \\ 4. Department of Thoracic Surgery, Xiangya Hospital, Central South University, Changsha, PR China; \\ 5. Institute of Medical Sciences, Xiangya Hospital, Central South University, Changsha, PR China; \\ 6. National Clinical Research Center for Geriatric Disorders, Xiangya Hospital, Central South University, Changsha, PR China. \\ $\llbracket$ Corresponding author: Chaojun Duan, Fax: 086-731-84327321; E-mail: duancjxy@126.com \\ (c) The author(s). This is an open access article distributed under the terms of the Creative Commons Attribution License (https://creativecommons.org/licenses/by/4.0/). \\ See http://ivyspring.com/terms for full terms and conditions.
}

Received: 2018.10.22; Accepted: 2019.09.18; Published: 2019.10.22

\begin{abstract}
Drug resistance is an important cause of failure in cancer chemotherapies. A large number of long noncoding RNAs (IncRNAs) have been found to be related to drug resistance in cancers. Therefore, IncRNAs provide potential targets for cancer therapies. The IncRNAs involved in cancer drug resistance are attracting interest from an increasing number of researchers. This review summarizes the latest research on the mechanisms and functions of IncRNAs in cancer drug resistance and envisages their future developments and therapeutic applications. This research suggests that IncRNAs regulate drug resistance through multiple mechanisms. LncRNAs do not affect drug resistance directly; usually, they do so by regulating the expression of some intermediate regulatory factors. In addition, IncRNAs exhibit a diversity of functions in cancer drug resistance. The overexpression of most IncRNAs promotes drug resistance, while a few IncRNAs have inhibitory effects.
\end{abstract}

Key words: IncRNA; cancer; drug resistance; chemotherapy; exosome

\section{Introduction}

Currently, cancer has become a major threat to human health with increasing mortality. Many cancer patients are in an advanced stage, and few of them are cured by operations. Chemotherapy, as the main treatment for middle-late cancers, can significantly enhance survival rates. However, drug resistance is a major obstacle to the success of cancer chemotherapies. Many studies have focused on finding effective therapeutic targets for cancer drug resistance. LncRNAs, which were previously regarded as transcriptional noise, have been found to be associated with gene expression regulation concerning drug resistance [1]. In addition, lncRNAs are also involved in cell proliferation, differentiation and tumor development.

It is widely accepted that only $2 \%$ of the human genome participates in protein coding, while the remaining $98 \%$ is transcribed into noncoding RNAs
(ncRNAs) [2, 3], which can be classified as small ncRNAs and lncRNAs based on their size. Small ncRNAs are those smaller than 200 nucleotides, such as microRNA (miRNA) and small interfering RNA (siRNA). LncRNAs are larger than 200 nucleotides and lack the important open reading frames [4]. LncRNAs are mostly distributed in the nucleus and are highly similar to protein-coding RNAs in the aspects of synthesis and processing. The similarity is attributable to the fact that Polymerase II also transcribes lncRNAs, which generally have fewer exons and lower expression levels than mRNAs. In addition, lncRNAs are more cell type specific and less conserved than mRNAs. Most lncRNAs contain a proximal promoter sequence, intronic or exonic sequences, and a secondary RNA structure [5].

The diversity of lncRNAs suggests potential multifunctions associated with normal physiological 
processes in cells. It has been reported that lncRNAs play a significant role in many life activities, such as dosage compensation effect, cell cycle regulation, epigenetic regulation and cell differentiation regulation [6]. Moreover, lncRNAs at different locations have different functions. For example, nuclear lncRNAs take part in transcriptional regulation, RNA processing and chromatin interaction [7]. Cytoplasmic lncRNAs are involved in the stability and translation of mRNAs and affect cell signaling [8].

Exploring the drug-resistant mechanisms and functions of lncRNAs in cancers has become a heavily researched subject in the bioscience field. It has been confirmed that ectopic expression of lncRNAs can result in malignant transformation and some common cancers, such as breast cancer, liver cancer and lung cancer, by interacting with DNA, RNA, and protein. However, although thousands of lncRNAs have been identified, their relationship with cancer drug resistance requires further research. In this review, we summarize the recent progress in research examining the role of lncRNAs in the drug resistance of cancer, including their mechanisms and functions, and discuss the future prospects of using lncRNAs as potential targets for cancer therapy.

\section{Mechanisms of cancer drug resistance related to IncRNAs}

Drug resistance can be roughly divided into intrinsic resistance and acquired resistance based on occurrence mechanisms [9, 10]. Intrinsic resistance means that resistance factors have already existed in cancer cells before chemotherapy, while acquired resistance results from gene mutations or the acquisition of exogenous resistance genes during treatment. Generally, drug resistance mechanisms that relate to tumor proliferation and prevention of tumor cell death include increased rates of drug efflux, apoptosis inhibition, alterations in the tumor microenvironment and metabolism, emergence of cancer stem cells, and mutations of drug targets [11]. In this section, the mechanisms of cancer drug resistance related to lncRNAs are summarized. Table 1 lists the IncRNAs that have recently been determined to be associated with cancer drug resistance and outlines their regulating targets/pathways.

\section{Promoting cell proliferation}

Since cell proliferation is closely associated with drug resistance, IncRNA LUCAT1 promotes methotrexate resistance in osteosarcoma by regulating the miR-200c/ABCB1 axis. In addition, LUCAT1 enhances the expression of the genes associated with drug resistance and promotes the proliferation and invasion of osteosarcoma cells [12]. In contrast to LUCAT1, lncRNA FENDRR sensitizes osteosarcoma cells to doxorubicin by suppressing $A B C B 1$ and ABCC1 [13]. Bian et al. found that UCA1 improves cell proliferation and drug resistance to 5-FU in CRC by suppressing miR-204-5p [45]. In addition, there is a UCA1-miR-204-5p-CREB1/BCL2/RAB22A regulatory network in CRC. Furthermore, lncRNA CRNDE promotes the proliferation and drug resistance of CRC cells by miR-181a-5p mediating Wnt/ $\beta$-catenin signaling. CRNDE also mediates LIMK2b by regulating the enhancer of zeste homolog 2 (EZH2), which promotes drug resistance in CRC [14].

\subsection{Regulating drug efflux}

Drug efflux alteration is the most common mechanism for cancer drug resistance. With increasing drug efflux, the intracellular drug concentration grows lower than the cell death threshold, thereby causing drug resistance. The expression levels of transporters, including ATP-binding cassette (ABC) protein, multidrug resistance associated protein (MRP) and copper transport protein (CTP), are related to drug efflux and are affected by various lncRNAs [15].

Downregulating the expression of lncRNA H19 lowers the cellular drug accumulation level in human hepatocellular carcinoma (HCC) by enhancing the methylation of the multidrug resistant gene 1 (MDR1) promoter and upregulating p-glycoprotein expression, which ultimately leads to doxorubicin resistance. H19 also affects drug resistance in breast cancer via the H19-CUL4A-ABCB1/MDR1 pathway. Experimental results showed that knocking out H19 significantly increased MDR1 and MRP4 in Dox-resistant breast cancer cells, which identified the role of H19 in breast cancer resistance [16].

LncRNAPVT1 was found to be highly expressed in tissues of cisplatin-resistant patients with gastric cancer. In addition, the results of qRT-PCR analysis and western blotting show that PVT1 upregulates the expression of MDR1, MRP, mTOR and HIF-1a, which promotes the development of MDR and suggests a potential target in gastric cancer therapy [17].

\subsection{Mutations of drug targets}

Drug-induced drug resistance is one of the major obstacles that may lead to treatment failure during tumor treatment. Various genetic changes occur when tumor cells divide. In a new generation of tumor cells, some cells may exhibit intrinsic resistance to specific chemotherapeutic drugs. In addition, some tumor cells may carry a gene that produces therapeutic drug-induced resistance [18]. 
Table 1. LncRNAs that have been or might be linked to cancer drug resistance

\begin{tabular}{|c|c|c|c|}
\hline Cancer type & LncRNAs & Drug resistance & Function \\
\hline \multirow[t]{7}{*}{ Breast cancer } & H19 [46] & Paclitaxel & Silencing of the pro-apoptotic gene BIK \\
\hline & UCA1 [55] & Doxorubicin & $\begin{array}{l}\text { Suppressing the p27 protein level and promoting cell growth and } \\
\text { tumorigenesis }\end{array}$ \\
\hline & HOTAIR [56] & Tamoxifen & $\begin{array}{l}\text { Interacting with ER and improving transcriptional activities of ER; } \\
\text { its overexpression upregulates breast carcinoma cell proliferation }\end{array}$ \\
\hline & CCAT2 [57] & Tamoxifen & Promoting cell migration \\
\hline & BCAR4 [58] & Tamoxifen & NA \\
\hline & ROR [59] & Tamoxifen & Induces autophagy \\
\hline & ATB [60] & Trastuzumab & Promotes cell proliferation, EMT, invasion and metastasis \\
\hline \multirow[t]{5}{*}{ Gastric cancer } & MRUL [61] & Vincristine & Enhancing ABCB1 expression \\
\hline & BCAR4 [62] & Cisplatin & NA \\
\hline & HNRNPC [63] & $\begin{array}{l}\text { 5-fluorouracil } \\
\text { Paclitaxel } \\
\text { Cisplatin }\end{array}$ & $\begin{array}{l}\text { Its high expression level indicates poor overall survival and free of } \\
\text { progression }\end{array}$ \\
\hline & AK002798 [64] & $\begin{array}{l}\text { Paclitaxel } \\
\text { Cisplatin }\end{array}$ & $\begin{array}{l}\text { Increasing cell apoptosis and the expression of P-glycoprotein and } \\
\text { MRP1; decreasing the expressions of Caspase } 3 \text { and Caspase } 8\end{array}$ \\
\hline & PVT1 [17] & Cisplatin & $\begin{array}{l}\text { Overexpression of LncRNA PVT1 in gastric carcinoma promotes } \\
\text { the development of MDR }\end{array}$ \\
\hline \multirow[t]{5}{*}{ Bladder cancer } & CUDR [65] & Cisplatin & Downregulating VEGF/PI3K/Akt signaling pathway \\
\hline & UCA1 [66] & Cisplatin/gemcitabine & Modulating miR-196a-5p of bladder carcinoma cells \\
\hline & NCRAN [67] & Cisplatin & Upregulating drug resistance; suppressing apoptosis \\
\hline & LET/NF90/miR-145 [68] & Gemcitabine & Upregulating TGF $\beta 1$ \\
\hline & GAS5 [69] & Doxorubicin & $\begin{array}{l}\text { Promoting apoptosis; depressing the expression of anti-apoptosis } \\
\text { protein Bcl-2 }\end{array}$ \\
\hline \multirow[t]{10}{*}{ Lung cancer } & HOTAIR & Crizotinib [70] & Activating autophagy by inhibiting the phosphorylation of ULK1 \\
\hline & & Gefitinib [71] & Activating Bax/Caspase-3 and TGF-a/EGFR signaling \\
\hline & AK126698 [72] & Cisplatin & $\begin{array}{l}\text { Suppressing canonical Wnt signaling pathway; downregulating } \\
\text { cisplatin resistance }\end{array}$ \\
\hline & MEG3 [41] & Cisplatin & $\begin{array}{l}\text { Enhancing cisplatin sensitivity via regulating miR-21-5p/SOX7 } \\
\text { axis; activating p53 and Bcl-xl of LAD cells }\end{array}$ \\
\hline & XIST [32] & Cisplatin & $\begin{array}{l}\text { Enhancing the drug resistance of NSCLC cells via suppressing } \\
\text { autophagy }\end{array}$ \\
\hline & AK001796 [73] & Cisplatin & Upregulating the expression of ANRIL \\
\hline & KCNQ1OT1 [74] & Paclitaxel & Upregulating the chemoresistance \\
\hline & MIAT [9] & Gefitinib & Regulating miR-34a \\
\hline & TUG1 [75] & Cisplatin & Regulating LIMK2b via EZH2 \\
\hline & ANRIL [76] & Cisplatin & Inhibits proliferation, induces apoptosis \\
\hline \multirow[t]{6}{*}{ Colorectal cancer } & H19 [77] & Doxorubicin & Overexpression of $\mathrm{H} 19$ activating Wnt/ $\beta$-catenin pathway \\
\hline & CUDR [78] & 5-fluorouracil & Upregulating miR-195 \\
\hline & & Oxaliplatin & Regulating Wnt/ $\beta$-catenin signaling via MiR-181a-5p \\
\hline & SNAR [79] & 5-Fluorouracil & Suppressing the drug resistance to 5 -FU \\
\hline & Lnc00152 [80] & Oxaliplatin & Functioning as a competing endogenous RNA \\
\hline & SLC25A25-AS1 [81] & Cisplatin & Downregulating SLC25A25-AS1 to promote proliferation \\
\hline \multirow[t]{4}{*}{ Hepatocellular carcinoma } & H19 [15] & Doxorubicin & $\begin{array}{l}\text { Inducing the expression of P-glycoprotein and the drug resistance } \\
\text { of MDR1; regulating MDR1 promoter methylation }\end{array}$ \\
\hline & Lnc-VLDLR [82] & $\begin{array}{l}\text { Sorafenib, } \\
\text { Camptothecin, } \\
\text { Doxorubicin }\end{array}$ & $\begin{array}{l}\text { Promoting cell-cycle progression; knocking out it suppresses } \\
\text { ABCG2 expression }\end{array}$ \\
\hline & HULC [35] & $\begin{array}{l}\text { Oxaliplatin, } \\
\text { 5-Fluorouracil } \\
\text { Pirarubicin }\end{array}$ & Stabilizing Sirt1 \\
\hline & ARSR [83] & Doxorubicin & $\begin{array}{l}\text { Promoting PTEN mRNA degradation; suppressing PTEN } \\
\text { expression; activating p13k/AKt pathway }\end{array}$ \\
\hline \multirow[t]{6}{*}{ Osteosarcoma } & LUCAT1 [84] & Methotrexate & Regulating drug resistance through miR-200c/ABCB1 axis \\
\hline & FENDRR [85] & Doxorubicin & Downregulating $\mathrm{ABCB} 1$ and $\mathrm{ABCC} 1$ \\
\hline & ODRUL [86] & Doxorubicin & $\begin{array}{l}\text { Downregulation of ODRUL partly suppresses the drug resistance to } \\
\text { doxorubicin }\end{array}$ \\
\hline & NR_036444 [87] & Doxorubicin & NA \\
\hline & FOXC2-AS1 [88] & Doxorubicin & Increases the expression of FOXC2 \\
\hline & Lnc00161 [89] & Cisplatin & Induces apoptosis regulating the miR-645-IFIT2 axis \\
\hline \multirow[t]{2}{*}{ Glioblastoma } & AC023115.3 [90] & Cisplatin & Reducing autophagy \\
\hline & MALAT1 [25] & Temozolomide & Promoting microRNA-101 \\
\hline Human squamous carcinoma & CUDR [91] & 5-Fluorouracil & $\begin{array}{l}\text { Causing a drug-induced apoptosis of cells by EMT and cancer stem } \\
\text { cell-like properties }\end{array}$ \\
\hline Cervical cancer & GAS5 [92] & Cisplatin & Suppressing tumor via microRNA 21, \\
\hline Cancer-associated fibroblasts & ANRIL [93] & Cisplatin & Upregulating the expression of ANRIL \\
\hline Squamous cell carcinoma & EGFR-AS1 [94] & Gefitinib & Mediating epidermal growth factor receptor addiction and \\
\hline
\end{tabular}




\begin{tabular}{|c|c|c|c|}
\hline Cancer type & LncRNAs & Drug resistance & Function \\
\hline & & & modulating treatment response \\
\hline Endometrial cancer & LncC00672 [95] & Paclitaxel & $\begin{array}{l}\text { Contributing to gene suppression mediated by p } 53 \text { protein and } \\
\text { promoting the chemosensitivity of endometrial cancer }\end{array}$ \\
\hline \multirow[t]{2}{*}{ Ovarian cancer } & ENST00000457645 [96] & Cisplatin & Modifying apoptotic protein expression \\
\hline & HOTAIR [97] & Carboplatin & DNA methylation \\
\hline Cholangiocarcinoma & NEAT-1 [98] & Gemcitabine & BAP1 overexpressing \\
\hline Nasopharyngeal carcinoma & N375709 [99] & Paclitaxel & NA \\
\hline Esophageal squamous cell carcinoma & AFAP1-AS1 [100] & Cisplatin & Upregulating \\
\hline Renal cancer & SRLR [101] & Sorafenib & Evokes IL-6/STAT3 axis \\
\hline
\end{tabular}

LncRNA BC087858 induces NSCLC resistance to EGFR-TKIS by a non-T790M mutation and activates the PI3K/AKT and MEK/ERK pathways and EMT [19]. LncRNA BC087858 knockout restored the sensitivity to gefitinib and suppressed the activation of the PI3K/AKT and MEK/ERK pathways and EMT by promoting the expression of ZEB1 and snails. At the same time, UCA1 expression was significantly upregulated in lung cancer patients with gefitinib resistance [20]. Furthermore, overexpression of UCA1 was associated with shorter survival in lung cancer. By activating the AKT/mTOR pathway and EMT, mTOR effectively changes the expression of UCA1, which restored gefitinib sensitivity in acquired resistance cells with non-T790M mutations.

Mutation is a non-negligible resistance mechanism. However, the relationship between lncRNA and mutations in drug resistance remains to be discovered.

\subsection{Arresting cell cycle}

DNA damage repair and warding off cell death induce cell cycle arrest, which allows repairing the damage. The mammalian cell cycle is precisely controlled by cyclin-dependent kinases (CDKs) and related pathways, such as the pRB and p53 pathways. In cancer, cell cycle arrest is disrupted by gain- and loss-of-function mutations, such as in p53 [21]. Recent studies have shown that many lncRNAs are involved in the regulation of key regulators of the cell cycle, such as cyclins, CDKs, CDK inhibitors, pRB and p53 [22]. These lncRNAs play a role in epigenetic regulation, transcription factor regulation, posttranscriptional regulation and protein scaffolding. These lncRNAs control the levels of cell cycle regulators through a variety of mechanisms, possibly providing diversity and reliability for the general cell cycle.

Current research indicates that CDKs can regulate cell cycle, cell proliferation and apoptosis, and other biological processes, but few studies have investigated CDK-related cell cycle arrest and drug resistance. CDKs and their associated pathways (pRB and p53) may represent a potential research direction in the field of drug resistance.

Interestingly, blocking CDK can induce cell cycle arrest, which reduces the curative effect of chemotherapy. It has been reported that inhibiting lncRNA NONHSAT028712 significantly reduces CDK2 mRNA levels and induces cell cycle arrest in G1 phase. In addition, receptor tyrosine kinase (RTK)/RAS signaling and cyclin E1 play important roles in CDK2 inhibitor resistance by activating E2F and ETS [23]. It can be inferred that the ETS factor ETV5 may be the downstream target of E2F.

\subsection{Inducing EMT}

Epithelial-mesenchymal transition (EMT) is a process in which epithelial cells are connected to the basement membrane, lose their polarity and intercellular adhesion ability, and ultimately become ectomesenchymal cells. This change in biological characteristics enables cells to migrate and invade. It is widely accepted that EMT is essential for stemness, tumor progression, metastasis and drug resistance. Chemotherapy has been shown to be a main inducer of EMT, eventually leading to secondary resistance in cancer cells. It has also been reported that lncRNA MALAT1 increases oxaliplatin resistance in CRC by promoting EMT [24, 25]. Suppressing the expression of lncRNA SLC25A25-AS1 promotes EMT and drug resistance in CRC [26]. This effect indicates that the curative effect of chemotherapy can also be enhanced by upregulating certain lncRNAs.

\subsection{Promoting glycometabolism}

Suppressing glycolysis significantly promotes apoptosis in drug-resistant cancer cells [27]. Fig. 1 shows four lncRNAs related to glycolysis: (1) lncRNA Ftx promotes the expression of peroxisome proliferator-activated receptor $\gamma$ (PPAR $\gamma$ ), and PPAR $\gamma$ upregulates the expression of enzymes in carbohydrate metabolism, which ultimately enhances aerobic glycolysis in HCC. In addition, the alterations in lactate production, glucose uptake and the enzyme expression induced by Ftx can be counteracted by downregulating the expression of PPARY [28]; (2) lncRNA UCA1 promotes glycolysis by upregulating hexokinase 2 (HK2) in cervical cancer [29]; (3) lncRNA-p23154 suppresses the expression of miR-378a-3p, and miR-378a-3p can promote 
Glut1-mediated glycolysis in oral squamous cell cancer by binding and inhibiting Glut1 [30]; and (4) lncRNA CRYBG3 increases the expression of lactate dehydrogenase A (LDHA), and LDHA promotes glycolysis in lung cancer. Furthermore, glycolysis can enhance cell proliferation [31]. Therefore, lncRNAs can be potential targets for cancer therapy due to their effects on glycolysis.

\section{Cell death}

\subsection{Dual regulation of autophagy}

Autophagy is the main cause of chemotherapeutic failure in non-small-cell lung cancer (NSCLC) and can be regulated by lncRNAs. For example, IncRNA XIST significantly decreases autophagy by regulating ATG7, which indicates that XIST may be a potential target for cisplatin chemotherapy of NSCLC. In addition, the XIST/miR-17/autophagy pathway may also be a promising target [32]. Besides, IncRNA AC023115.3 suppresses drug resistance in glioblastoma by inhibiting autophagy [33].

Autophagy has dual characteristics of tumor suppressor and carcinogen and is regulated by various signaling pathways, such as PI3K-Akt-m TOR, Beclin1, BCL-2, Ras and p53. A recent study showed that the overexpression of IncRNA HOTAIR increases drug resistance in NSCLC by promoting autophagy through inhibiting the phosphorylation of ULK1. Similarly, upregulating lncRNA CASC2 can sensitize glioma to the cytotoxicity of temozolomide by suppressing autophagy [34]. However, IncRNA XIST increases chemotherapy resistance in NSCLC cells by inhibiting autophagy [32]. In addition, lncRNA HULC induces autophagy in HCC by stabilizing silent information regulator 1 (Sirt1). As a result, this lncRNA enhances drug resistance to three anti-cancer drugs: oxaliplatin, pirarubicin and 5-FU. It is also revealed that the drug resistance in $\mathrm{HCC}$ can be increased through the HULC/USP22/Sirt1/protective autophagy pathway [35].

\subsection{Suppressing apoptosis}

Since most drugs prevent cancer cell growth by promoting apoptosis, suppressing apoptosis may induce drug resistance and accelerate the proliferation of cancer cells. It has been reported that lncRNAs can increase apoptosis-induced drug resistance by upregulating survival factors, such as BCL-2, nuclear factor kappa B, and inhibitor of apoptosis protein [36]. Additionally, lncRNA HOTTIP enhances drug resistance in small cell lung cancer (SCLC) by promoting BCL-2 expression [37]. Moreover, UCA1 increases cisplatin resistance by upregulating Wnt6 expression and suppressing cell apoptosis in bladder cancer [38].

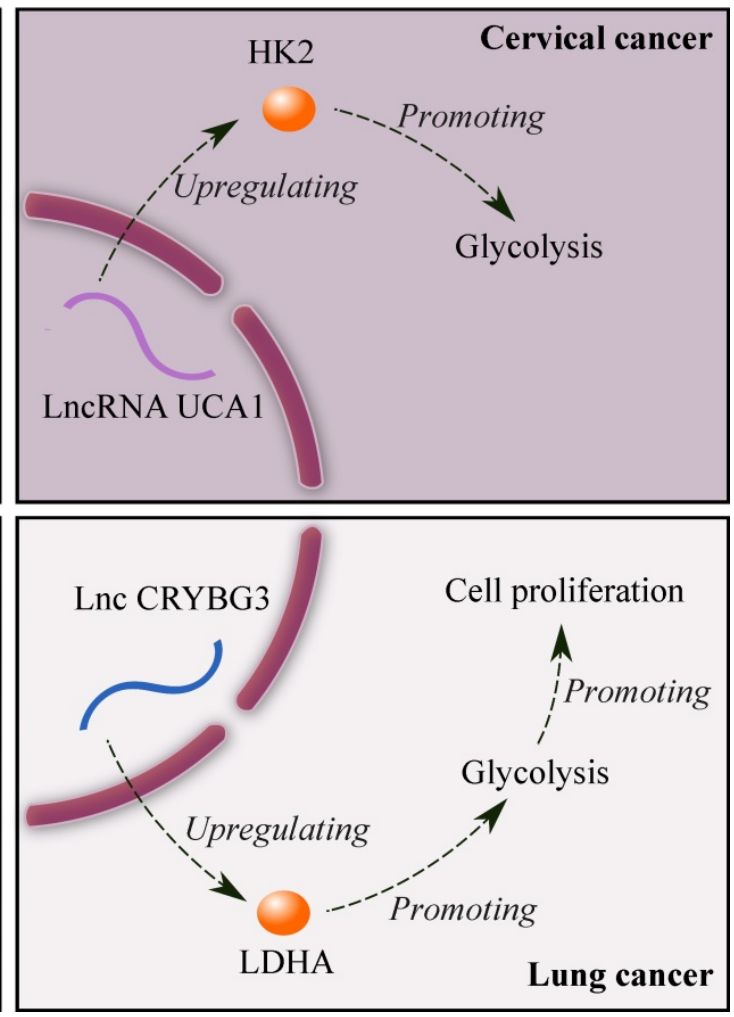

Figure 1. Mechanisms of glycolysis regulated by four IncRNAs. Symbols: brown ellipses - enzymes; brown Y shape - receptor (PPARY). 
The overexpression of HOTAIR improves gastric cancer cell proliferation and cell cycle G1/S transition. In addition, HOTAIR overexpression decreases cancer cell apoptosis, which can activate the P13K/AKT/MRP1 genes by targeting miR-126. As a result, cisplatin resistance in gastric cancer is increased [39]. LncRNA GAS5 is closely associated with doxorubicin resistance in bladder transitional cell cancer (BTCC) and inhibiting proliferation. The expression of this lncRNA is suppressed in UBC and increases with the pathological grades of BTCC. The overexpression of GAS5 promotes apoptosis by decreasing the expression of the anti-apoptosis protein BCL-2. Similarly, UCA1 promotes cisplatin resistance in bladder cancer by increasing the expression of Wnt6 [40].

Besides, lncRNA MEG3 regulates cisplatin (DDP) resistance in NSCLC by acting as a competing endogenous RNA. MEG3 can interact with miR-21-5p directly and decrease its expression, and miR-21-5p can significantly eliminate the effects of MEG3 on DDP resistance by regulating cell proliferation and apoptosis. Moreover, SOX7 is a direct target for MEG3 and miR-21-5p and upregulating the miR-21-5p/ SOX7 axis can reverse the pro-proliferative and anti-apoptotic effects induced by knocking out MEG3. This finding helps to elucidate the molecular mechanism of DDP resistance associated with MEG3 in NSCLC [41].

MEG3 promotes chemosensitivity by enhancing apoptosis induced by oxaliplatin. However, Li et al. found that lncRNA TUG1 works as a competing endogenous RNA (ceRNA) to sponge miR-186, and miR-186 targets CPEB2 directly in CRC [42].

The overexpression of H19 can increase methotrexate resistance in colorectal cancer by inducing EMT. In addition, lncRNA PVT1 promotes EMT by upregulating the expression of the transcription factor Twist1 through the sponge effect [43].

\subsection{Microenvironmental changes}

Microenvironmental changes can lead to drug resistance in cancers. It was found that the large oxygen consumption of cancer cells causes hypoxic microenvironments in solid tumors. Therefore, cancer cells switch to anaerobic respiration, which results in persistent acidic microenvironments. Hypoxic and acidic microenvironments can lead to genetic instability and activate signaling pathways [44]. Emerging evidence shows that exosomes affect tumor-associated pathways in microenvironments, including angiogenesis, cancer stemness, cell metastasis and EMT driven by hypoxia [45]. For example, as shown in Fig. 2 (A1 A4), exosomes secrete proteins and mRNAs, which induce a desmoplastic reaction (DR) and promote fibroblast growth. As a result, the circulation of anticancer drugs is blocked, and drug resistance is acquired. Furthermore, since tumor nodes are often not close to capillaries, tumor microenvironments are limited in oxygen and essential nutrients. This limitation upregulates autophagy and suppresses cell proliferation, which may contribute to the resistance to cycle-active anticancer drugs.

Exosomes promote drug resistance to tamoxifen in breast cancer by regulating the transfer of UCA1. The exosomes from tamoxifen-resistant cells contain notably more UCA1s than those from tamoxifen-sensitive cells. Knocking out UCA1 can decrease tamoxifen resistance mediated by exosomes. In addition, H19 regulates drug resistance in ERa-positive breast cancer by epigenetically silencing the pro-apoptotic gene BIK [46]. As illustrated in Fig. 2 (B1 B3), the exosomes released from breast cancer cells increase the expression of UCA1, which enhances cell viability. Consequently, apoptosis is suppressed, and drug resistance can be increased.

\section{Other mechanisms}

\subsection{Cancer stemness}

The expression of lncRNA LET is suppressed in urinary bladder cancer (UBC) after gemcitabine treatment. The overexpression of TGF $\beta 1$ and a low level of lncRNA LET and miR-145 can predict poor prognosis. TGF $\beta 1$ also promotes gemcitabine resistance by downregulating the signaling of the LET/NF90/miR-145 axis. In addition, TGF $\beta 1$ can increase drug resistance in UBC by promoting cancer stemness [47].

\subsection{Epigenetic modification}

Recent studies have shown that lncRNAs are greatly involved in drug resistance by epigenetic modification, especially methylation. For example, lncRNA HOTAIR suppresses HOXA1 methylation by inhibiting DNMT1 and DNMT3B, which leads to chemoresistance in SCLC [48]. H19 promotes p-glycoprotein overexpression and drug resistance in HCC by regulating the promoter methylation of MDR1 [49]. It is worthwhile to investigate the relationship between drug resistance and gene methylation affected by lncRNAs. LncRNA can affect cell proliferation by mediating methylation in pancreatic cancer. For example, lncRNA HOTAIR regulates EZH2 and miR-34a by mediating the methylation of H3K27 and suppresses the miR-663b promoter by altering histone methylation, and EZH2, miR-34a and miR-663b can affect cell proliferation [50]. 


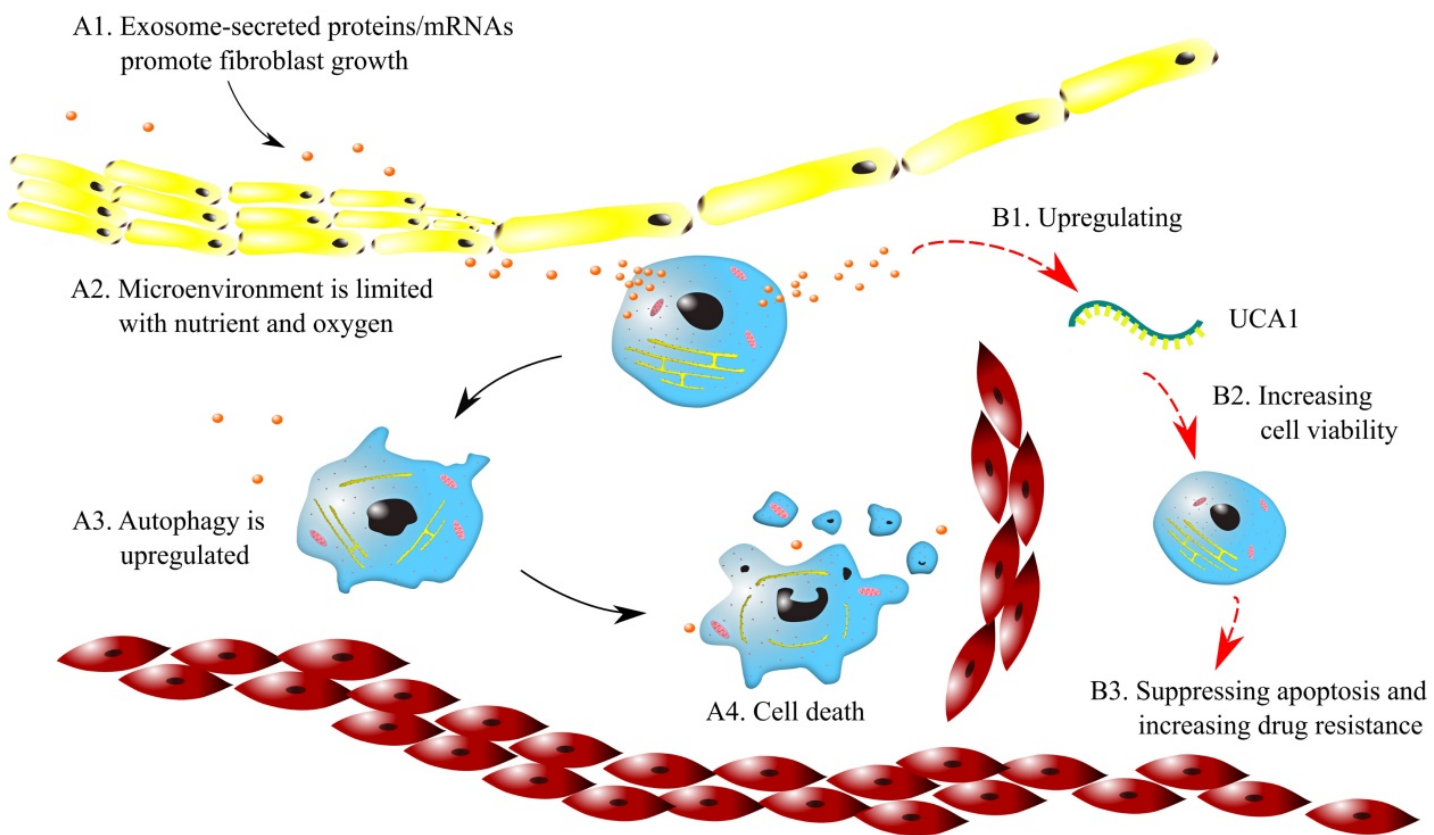

Figure 2. Mechanisms of drug resistance regulated by exosomes in microenvironments. Symbols: red cells - red blood cells; blue cells - cancer cells; yellow cells fibroblast; brown dots - exosomes; green curve - IncRNA UCA1; black arrows - for A1 A4; red arrows - for B1 B3.

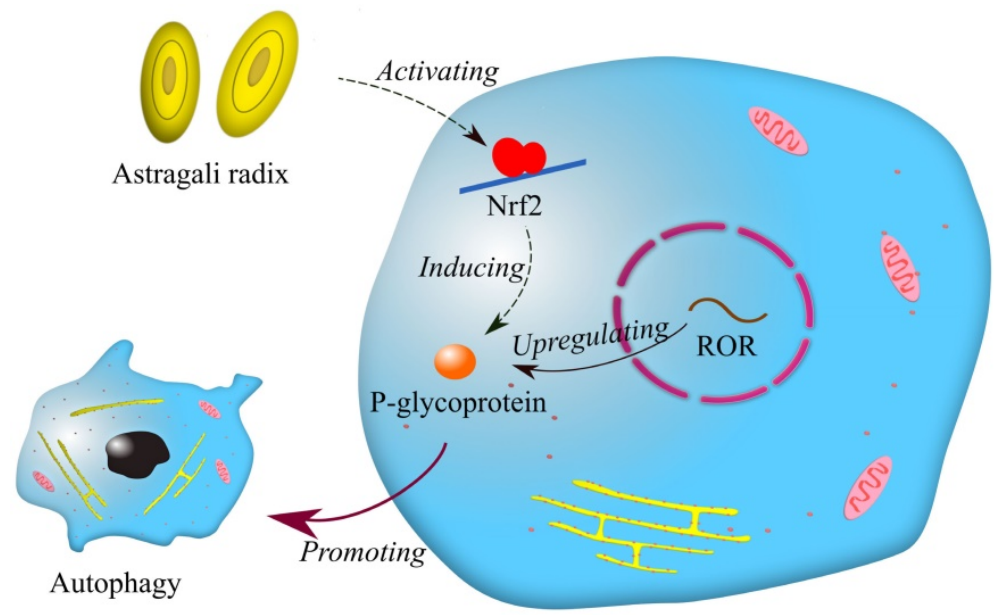

Figure 3. Mechanisms of drug resistance regulated by astragali radix and IncRNA ROR. Symbols: blue cells—breast cancer cells; red circle—nuclear membranes; brown dots—enzymes; yellow net—endoplasmic reticulum; pink ellipses—mitochondria.

Phosphorylation is an important factor for drug resistance based on genetic modification. The overexpression of HOTAIR reduces the sensitivity to cisplatin by activating STAT3 and increasing the expression of ABCB1. In addition, promoting the phosphorylation of STAT3 suppresses ABCB1 directly, which results in chemoresistance to cisplatin in HCC [51]. Further research is warranted to determine the mechanisms of cancer drug resistance associated with phosphorylation.

\subsection{Chinese herbal medicines}

In the last ten years, extracts from Chinese herbal plants, as a natural addition, have been commonly employed in the development of new anti-cancer drugs because of their mildness and effectiveness. It has been reported that p-glycoprotein is associated with multidrug resistance in breast cancer [52]. Afterwards, $\mathrm{Li}$ et al. found that IncRNA ROR increases the expression of p-glycoprotein, and p-glycoprotein is involved in drug resistance in breast cancer by promoting autophagy [53]. Interestingly, a recent report showed that astragali radix promotes the expression of p-glycoprotein by activating Nrf2 [54]. It can be inferred that astragali radix may be related to lncRNA ROR in drug resistance of breast cancer (Fig. 3). Astragali radix is a famous Chinese herbal medicine widely used in traditional Chinese medicine prescriptions. Although it was previously believed that traditional Chinese medicine had little effect on treating cancers, it is now reasonable to think 
that traditional Chinese medicine may benefit cancer therapy by regulating drug resistance.

\section{Conclusions}

This review summarizes the drug-resistance mechanisms of lncRNAs in cancers. The review contains not only the typical mechanisms, that is, drug efflux, mutations of drug targets, arresting cell cycle, EMT, autophagy and apoptosis, but also newly discovered mechanisms, including glycolmetabolism, microenvironmental changes, cancer stemness, genetic modification and Chinese herbal medicines. Obtained data indicate that lncRNAs are involved in cancer drug resistance by regulating some intermediate regulatory factors. In addition, this review lists many lncRNAs that have recently been found to be related to cancers and their regulatory pathways. The functions of IncRNAs in cancer drug resistance are described in detail according to cancer types. A certain lncRNA can regulate the drug resistance of multiple cancers, and a certain kind of cancer can be associated with several lncRNAs. Finally, the overexpression of most lncRNAs enhances drug resistance in cancers, while a few of them can sensitize the cells to anticancer drugs. This effect demonstrates the functional diversity of lncRNAs, which requires further research. In addition, it is necessary to further elucidate the functions of more lncRNAs in cancer drug resistance and their mechanisms, which will provide more targets for cancer therapies.

\section{Future prospects}

Although many studies have focused on the functions and mechanisms of lncRNAs in cancer drug resistance, a large proportion of them remain unrevealed and warrant further investigation. Future drugs for cancer chemotherapies can be combinations of existing anticancer drugs and drugs targeting lncRNAs related to drug resistance. Besides, we anticipate that some studies can be conducted to identify the basic drug-resistant mechanisms associated with lncRNAs, which may provide some common targets for cancer therapies. Moreover, the finding that astragali radix is involved in cancer drug resistance may lead to more research on the relations of traditional Chinese medicines and the drug resistance related to lncRNAs.

\section{Abbreviations}

LncRNAs: long noncoding RNAs; ncRNAs: noncoding RNAs; miRNA: microRNA; siRNA: small interfering RNA; MRP: multidrug resistance associated protein; ABC: ATP-binding cassette; CTP: copper transport protein; HCC: hepatocellular carcinoma; MDR1: multidrug resistant gene 1; CDKs: cyclin-dependent kinases; EMT: epithelialmesenchymal transition; PPARY: peroxisome proliferator-activated receptor $\gamma$; HK2: hexokinase 2; LDHA: lactate dehydrogenase A; NSCLC: non-smallcell lung cancer; Sirt1: silent information regulator 1; SCLC: small cell lung cancer; BTCC: bladder transitional cell cancer; DDP: cisplatin; ceRNA: competing endogenous RNA; DR: desmoplastic reaction; UBC: urinary bladder cancer.

\section{Acknowledgements}

This work was supported by the National Natural Science Foundation of China (No.81572281 and No.81702278). And we also thank Dr. Suiyu Chen for the helpful discussion.

\section{Competing Interests}

The authors have declared that no competing interest exists.

\section{References}

1. Peng Z, Liu C, Wu M. New insights into long noncoding RNAs and their roles in glioma. Mol Cancer. 2018; 17(1): 61.

2. Wu Q, Xiang $\mathrm{S}$, Ma J, et al. Long non-coding RNA CASC15 regulates gastric cancer cell proliferation, migration and epithelial mesenchymal transition by targeting CDKN1A and ZEB1. Mol Oncol. 2018; 28(Feb).

3. Zhao W, Liu Y, Zhang C, et al. Multiple Roles of Exosomal Long Noncoding RNAs in Cancers. Biomed Res Int. 2019; 2019: 1460572.

4. Rao MRS. Long Non Coding RNA Biology: Springer; 2017.

5. Wang C, Wang L, Ding Y, et al. LncRNA Structural Characteristics in Epigenetic Regulation. Int J Mol Sci. 2017; 18(12): 2659.

6. Jandura A, Krause HM. The New RNA World: Growing Evidence for Long Noncoding RNA Functionality. Trends Genet. 2017; 33(10): 665-676.

7. Chujo T, Hirose T. Nuclear Bodies Built on Architectural Long Noncoding RNAs: Unifying Principles of Their Construction and Function. Mol Cells. 2017; 40(12): 889-896.

8. Noh JH, Kim KM, McClusky WG, et al. Cytoplasmic functions of long noncoding RNAs. Wiley Interdisciplinary Reviews: RNA. 2018; 9(3): e1471.

9. Fu Y, Li C, Luo Y, et al. Silencing of Long Non-coding RNA MIAT Sensitizes Lung Cancer Cells to Gefitinib by Epigenetically Regulating miR-34a. Front Pharmacol. 2018; 9(Feb): 82 .

10. $\mathrm{Hu} \mathrm{Y}, \mathrm{Zhu} \mathrm{QN}$, Deng JL, et al. Emerging role of long non-coding RNAs in cisplatin resistance. Onco Targets Ther. 2018; 11: 3185-3194.

11. Hanahan D, Weinberg RA. Hallmarks of cancer: the next generation. Cell. 2011; 144(5): 646-674

12. Han Z, Shi L. Long non-coding RNA LUCAT1 modulates methotrexate resistance in osteosarcoma via miR-200c/ABCB1 axis. Biochem Bioph Res Co. 2018; 495(1): 947-953.

13. Kun-Peng $Z$, Xiao-Long $M$, Chun-Lin Z. LncRNA FENDRR sensitizes doxorubicin-resistance of osteosarcoma cells through down-regulating ABCB1 and ABCC1. Oncotarget. 2017; 8(42): 71881-71893.

14. Li C, Gao Y, Li Y, et al. TUG1 mediates methotrexate resistance in colorectal cancer via miR-186/CPEB2 axis. Biochem Bioph Res Co. 2017; 491(2): 552-557.

15. Tsang WP, Kwok TT. Riboregulator H19 induction of MDR1-associated drug resistance in human hepatocellular carcinoma cells. Oncogene. 2007; 26(33): 4877-4881.

16. Zhu Q, Wang G, Guo Y, et al. LncRNA H19 is a major mediator of doxorubicin chemoresistance in breast cancer cells through a cullin4A-MDR1 pathway. Oncotarget. 2017; 8(54): 91990-92003.

17. Zhang XW, Bu P, Liu L, et al. Overexpression of long non-coding RNA PVT1 in gastric cancer cells promotes the development of multidrug resistance. Biochem Biophys Res Commun. 2015; 462(3): 227-232

18. Feizabadi MS. Modeling multi-mutation and drug resistance: analysis of some case studies. Theor Biol Med Model. 2017; 14(1): 6 .

19. Pan H, Jiang $\mathrm{T}$, Cheng $\mathrm{N}$, et al. Long non-coding RNA BC087858 induces non-T790M mutation acquired resistance to EGFR-TKIs by activating $\mathrm{PI} 3 \mathrm{~K} / \mathrm{AKT}$ and MEK/ERK pathways and EMT in non-small-cell lung cancer. Oncotarget. 2016; 7(31): 49948-49960.

20. Cheng N, Cai W, Ren S, et al. Long non-coding RNA UCA1 induces non-T790M acquired resistance to EGFR-TKIs by activating the AKT/mTOR pathway in EGFR-mutant non-small cell lung cancer. Oncotarget. 2015; 6(27): 23582-23593. 
21. Nussinov R, Tsai CJ, Jang H. A New View of Pathway-Driven Drug Resistance in Tumor Proliferation. Trends Pharmacol Sci. 2017; 38(5): 427-437.

22. Kitagawa $M$, Kitagawa $K$, Kotake $Y$, et al. Cell cycle regulation by long non-coding RNAs. Cell Mol Life Sci. 2013; 70(24): 4785-4794.

23. Taylor-Harding B, Aspuria PJ, Agadjanian H, et al. Cyclin E1 and RTK/RAS signaling drive CDK inhibitor resistance via activation of E2F and ETS. Oncotarget. 2015; 6(2): 696-714.

24. Li P, Zhang X, Wang H, et al. MALAT1 Is Associated with Poor Response to Oxaliplatin-Based Chemotherapy in Colorectal Cancer Patients and Promotes Chemoresistance through EZH2. Mol Cancer Ther. 2017; 16(4): 739-751.

25. Cai T, Liu Y, Xiao J. Long noncoding RNA MALAT1 knockdown reverses chemoresistance to temozolomide via promoting microRNA-101 in glioblastoma. Cancer Med. 2018; 7(4): 1404-1415.

26. Li Y, Huang S, Li Y, et al. Decreased expression of LncRNA SLC25A25-AS1 promotes proliferation, chemoresistance, and EMT in colorectal cancer cells. Tumour Biol. 2016; 37(10): 14205-14215.

27. Cheng $\mathrm{C}$, Xie Z, Li Y, et al. PTBP1 knockdown overcomes the resistance to vincristine and oxaliplatin in drug-resistant colon cancer cells through regulation of glycolysis. Biomed Pharmacother. 2018; 108: 194-200.

28. Li X, Zhao Q, Qi J, et al. IncRNA Ftx promotes aerobic glycolysis and tumor progression through the PPARgamma pathway in hepatocellular carcinoma. Int J Oncol. 2018; 53(2): 551-566.

29. Fan L, Huang C, Li J, et al. Long noncoding RNA urothelial cancer associated 1 regulates radioresistance via the hexokinase 2/glycolytic pathway in cervical cancer. Int J Mol Med. 2018; 42(4): 2247-2259.

30. Wang $Y$, Zhang $X$, Wang Z, et al. LncRNA-p23154 promotes the invasion-metastasis potential of oral squamous cell carcinoma by regulating Glut1-mediated glycolysis. Cancer Lett. 2018; 434: 172-183.

31. Chen $\mathrm{H}$, Pei $\mathrm{H}, \mathrm{Hu} \mathrm{W}$, et al. Long non-coding RNA CRYBG3 regulates glycolysis of lung cancer cells by interacting with lactate dehydrogenase A. J Cancer. 2018; 9(14): 2580-2588.

32. Sun $\mathrm{W}, \mathrm{Zu} \mathrm{Y}, \mathrm{Fu} \mathrm{X}$, et al. Knockdown of IncRNA-XIST enhances the chemosensitivity of NSCLC cells via suppression of autophagy. Oncol Rep. 2017; 38(6): 3347-3354.

33. Ma B, Yuan Z, Zhang L, et al. Long non-coding RNA AC023115.3 suppresses chemoresistance of glioblastoma by reducing autophagy. Biochimica et Biophysica Acta (BBA) - Molecular Cell Research. 2017; 1864(8): 1393-1404.

34. Jiang C, Shen F, Du J, et al. Upregulation of CASC2 sensitized glioma to temozolomide cytotoxicity through autophagy inhibition by sponging miR-193a-5p and regulating mTOR expression. Biomed Pharmacother. 2018; 97(Jan): 844-850.

35. Xiong $\mathrm{H}, \mathrm{Ni} \mathrm{Z}, \mathrm{He}$ J, et al. LncRNA HULC triggers autophagy via stabilizing Sirt1 and attenuates the chemosensitivity of HCC cells. Oncogene. 2017; 36(25): 3528-3540.

36. Majidinia M, Yousefi B. Long non-coding RNAs in cancer drug resistance development. DNA Repair (Amst). 2016; 45: 25-33.

37. Sun $Y$, Hu B, Wang Q, et al. Long non-coding RNA HOTTIP promotes BCL-2 expression and induces chemoresistance in small cell lung cancer by sponging miR-216a. Cell Death Dis. 2018; 9(2): 85

38. Fan $Y$, Shen $B$, Tan $M$, et al. Long non-coding RNA UCA1 increases chemoresistance of bladder cancer cells by regulating Wnt signaling. Febs J. 2014; 281(7): 1750-1758.

39. Yan J, Dang Y, Liu S, et al. LncRNA HOTAIR promotes cisplatin resistance in gastric cancer by targeting miR-126 to activate the PI3K/AKT/MRP1 genes. Tumor Biology. 2016; 37(12): 16345-16355.

40. Fan $Y$, Shen B, Tan M, et al. Long non-coding RNA UCA1 increases chemoresistance of bladder cancer cells by regulating Wnt signaling. Febs J. 2014; 281(7): 1750-1758.

41. Wang $\mathrm{P}$, Chen $\mathrm{D}, \mathrm{Ma} \mathrm{H}$, et al. LncRNA MEG3 enhances cisplatin sensitivity in non-small cell lung cancer by regulating miR-21-5p/SOX7 axis. Onco Targets Ther. 2017; 10(Oct): 5137-5149.

42. $\mathrm{Li} \mathrm{C}, \mathrm{Gao}$ Y, Li Y, et al. TUG1 mediates methotrexate resistance in colorectal cancer via miR-186/CPEB2 axis. Biochem Bioph Res Co. 2017; 491(2): 552-557.

43. Chang Z, Cui J, Song Y. Long noncoding RNA PVT1 promotes EMT via mediating microRNA-186 targeting of Twist1 in prostate cancer. Gene. 2018; 654(May): 36-42.

44. Li W, Sun X. Recent advances in developing novel anti-cancer drugs targeting tumor hypoxic and acidic microenvironments. Recent Pat Anticancer Drug Discov. 2018.

45. Zhang C, Ji Q, Yang Y, et al. Exosome: Function and Role in Cancer Metastasis and Drug Resistance. Technol Cancer Res T. 2018; 17(Jan): 1180787079.

46. Si X, Zang R, Zhang E, et al. LncRNA H19 confers chemoresistance in ER $\alpha$ -positive breast cancer through epigenetic silencing of the pro-apoptotic gene BIK. Oncotarget. 2016; 7(49): 81452-81462.

47. Zhuang J, Shen L, Yang L, et al. TGF $\beta 1$ Promotes Gemcitabine Resistance through Regulating the LncRNA-LET/NF90/miR-145 Signaling Axis in Bladder Cancer. Theranostics. 2017; 7(12): 3053-3067.

48. Fang S, Gao H, Tong $\mathrm{Y}$, et al. Long noncoding RNA-HOTAIR affects chemoresistance by regulating HOXA1 methylation in small cell lung cancer cells. Lab Invest. 2016; 96(1): 60-68.

49. Tsang WP, Kwok TT. Riboregulator H19 induction of MDR1-associated drug resistance in human hepatocellular carcinoma cells. Oncogene. 2007; 26(33): $4877-4881$.
50. $\mathrm{Li} \mathrm{CH}, \mathrm{Xiao} \mathrm{Z}$, Tong $\mathrm{JH}$, et al. EZH2 coupled with HOTAIR to silence MicroRNA-34a by the induction of heterochromatin formation in human pancreatic ductal adenocarcinoma. Int J Cancer. 2017; 140(1): 120-129.

51. Zhou JJ, Cheng D, He XY, et al. Knockdown of long non-coding RNA HOTAIR sensitizes hepatocellular carcinoma cell to cisplatin by suppressing the STAT3/ABCB1 signaling pathway. Oncol Lett. 2017; 14(6): 7986-7992.

52. Zhang F, Zhang H, Wang Z, et al. P-glycoprotein associates with Anxa2 and promotes invasion in multidrug resistant breast cancer cells. Biochem Pharmacol. 2014; 87(2): 292-302.

53. Li Y, Jiang B, Zhu H, et al. Inhibition of long non-coding RNA ROR reverses resistance to Tamoxifen by inducing autophagy in breast cancer. Tumour Biol. 2017; 39(6): 1393383874

54. Lou Y, Guo Z, Zhu Y, et al. Astragali radix and its main bioactive compounds activate the Nrf2-mediated signaling pathway to induce P-glycoprotein and breast cancer resistance protein. J Ethnopharmacol. 2018.

55. Liu H, Wang G, Yang L, et al. Knockdown of Long Non-Coding RNA UCA1 Increases the Tamoxifen Sensitivity of Breast Cancer Cells through Inhibition of Wnt/ $\beta$-Catenin Pathway. Plos One. 2016; 11(12): e168406.

56. Xue X, Yang YA, Zhang A, et al. LncRNA HOTAIR enhances ER signaling and confers tamoxifen resistance in breast cancer. Oncogene. 2016; 35(21): 2746-2755.

57. Cai Y, He J, Zhang D. Suppression of long non-coding RNA CCAT2 improves tamoxifen-resistant breast cancer cells' response to tamoxifen. Mol Biol (Mosk). 2016; 50(5): 821-827.

58. Godinho MFE, Sieuwerts AM, Look MP, et al. Relevance of BCAR4 in tamoxifen resistance and tumour aggressiveness of human breast cancer. Brit J Cancer. 2010; 103(8): 1284-1291.

59. Zhang $\mathrm{H}$, Liang $\mathrm{F}$, Zhang J, et al. Effects of long noncoding RNA-ROR on tamoxifen resistance of breast cancer cells by regulating microRNA-205. Cancer Chemoth Pharm. 2017; 79(2): 327-337.

60. Shi S, Wang L, Yu B, et al. LncRNA-ATB promotes trastuzumab resistance and invasion-metastasis cascade in breast cancer. Oncotarget. 2015; 6(13): $11652-11663$

61. Wang $\mathrm{Y}$, Zhang $\mathrm{D}, \mathrm{Wu} \mathrm{K}$, et al. Long Noncoding RNA MRUL Promotes ABCB1 Expression in Multidrug-Resistant Gastric Cancer Cell Sublines. Mol Cell Biol. 2014; 34(17): 3182-3193.

62. Godinho MFE, Sieuwerts AM, Look MP, et al. Relevance of BCAR4 in tamoxifen resistance and tumour aggressiveness of human breast cancer. Brit J Cancer. 2010; 103(8): 1284-1291.

63. Huang $\mathrm{H}$, Han $\mathrm{Y}$, Zhang $\mathrm{C}$, et al. HNRNPC as a candidate biomarker for chemoresistance in gastric cancer. Tumor Biology. 2016; 37(3): 3527-3534.

64. Hang $Q$, Sun $R$, Jiang $C$, et al. Notch 1 promotes cisplatin-resistant gastric cancer formation by upregulating lncRNA AK022798 expression. Anti-Cancer Drug. $2015 ; 26(6): 1$.

65. Liao X, Tao L, Liu J, et al. Matrine combined with cisplatin synergistically inhibited urothelial bladder cancer cells via down-regulating VEGF/PI3K/Akt signaling pathway. Cancer Cell Int. 2017; 17(1): 124.

66. Pan $\mathrm{J}, \mathrm{Li} X, \mathrm{Wu}, \mathrm{W}$, et al. Long non-coding RNA UCA1 promotes cisplatin/gemcitabine resistance through CREB modulating miR-196a-5p in bladder cancer cells. Cancer Lett. 2016; 382(1): 64-76.

67. Zhu Y, Yu M, Li Z, et al. NcRAN, a Newly Identified Long Noncoding RNA, Enhances Human Bladder Tumor Growth, Invasion, and Survival. Urology. 2011; 77(2): 510-511.

68. Zhuang J, Shen L, Yang L, et al. TGF $\beta 1$ Promotes Gemcitabine Resistance through Regulating the LncRNA-LET/NF90/miR-145 Signaling Axis in Bladder Cancer. Theranostics. 2017; 7(12): 3053-3067.

69. Zhang H, Guo Y, Song Y, et al. Long noncoding RNA GAS5 inhibits malignant proliferation and chemotherapy resistance to doxorubicin in bladder transitional cell carcinoma. Cancer Chemother Pharmacol. 2017; 79(1): 49-55.

70. Yang Y, Jiang C, Yang Y, et al. Silencing of LncRNA-HOTAIR decreases drug resistance of Non-Small Cell Lung Cancer cells by inactivating autophagy via suppressing the phosphorylation of ULK1. Biochem Biophys Res Commun. 2018; 497(4): 1003-1010.

71. Liu $\mathrm{Y}$, Jiang $\mathrm{H}$, Zhou $\mathrm{H}$, et al. Lentivirus-mediated silencing of HOTAIR IncRNA restores gefitinib sensitivity by activating Bax/Caspase-3 and suppressing TGF-a/EGFR signaling in lung adenocarcinoma. Oncol Lett. 2017; 15(3): 2829-2838.

72. Yang Y, Li H, Hou S, et al. The Noncoding RNA Expression Profile and the Effect of lncRNA AK126698 on Cisplatin Resistance in Non-Small-Cell Lung Cancer Cell. Plos One. 2013; 8(5): e65309.

73. Liu B, Pan C, Ma T, et al. Long non-coding RNA AK001796 contributes to cisplatin resistance of non-small cell lung cancer. Mol Med Rep. 2017; 16(4): $4107-4112$

74. Ren K, Xu R, Huang J, et al. Knockdown of long non-coding RNA KCNQ1OT1 depressed chemoresistance to paclitaxel in lung adenocarcinoma. Cancer Chemoth Pharm. 2017; 80(2): 243-250.

75. Niu Y, Ma F, Huang W, et al. Long non-coding RNA TUG1 is involved in cell growth and chemoresistance of small cell lung cancer by regulating LIMK2b via EZH2. Mol Cancer. 2017; 16(1): 5

76. Gong W, Yin J, Li X, et al. Association of well-characterized lung cancer lncRNA polymorphisms with lung cancer susceptibility and platinum-based chemotherapy response. Tumor Biology. 2016; 37(6): 8349-8358.

77. Wu K, Liang $\mathrm{W}$, Feng L, et al. H19 mediates methotrexate resistance in colorectal cancer through activating Wnt/ $\beta$-catenin pathway. Exp Cell Res. 2017; 350(2): 312-317. 
78. Kong D, Zhang D, Chu X, et al. Schizandrin A enhances chemosensitivity of colon carcinoma cells to 5-fluorouracil through up-regulation of miR-195. Biomed Pharmacother. 2018; 99(Mar): 176-183.

79. Lee $\mathrm{H}, \mathrm{Kim} \mathrm{C}, \mathrm{Ku}$ J, et al. A Long Non-Coding RNA snaR Contributes to 5-Fluorouracil Resistance in Human Colon Cancer Cells. Mol Cells. 2014; 37(7): 540-546.

80. Yue B, Cai D, Liu C, et al. Linc00152 Functions as a Competing Endogenous RNA to Confer Oxaliplatin Resistance and Holds Prognostic Values in Colon Cancer. Mol Ther. 2016; 24(12): 2064-2077.

81. Li Y, Huang S, Li Y, et al. Decreased expression of LncRNA SLC25A25-AS1 promotes proliferation, chemoresistance, and EMT in colorectal cancer cells. Tumor Biology. 2016; 37(10): 14205-14215.

82. Takahashi K, Yan IK, Wood J, et al. Involvement of Extracellular Vesicle Long Noncoding RNA (linc-VLDLR) in Tumor Cell Responses to Chemotherapy. Mol Cancer Res. 2014; 12(10): 1377-1387.

83. Li Y, Ye Y, Feng B, et al. Long Noncoding RNA IncARSR Promotes Doxorubicin Resistance in Hepatocellular Carcinoma via Modulating PTEN-PI3K/Akt Pathway. J Cell Biochem. 2017; 118(12): 4498-4507.

84. Han Z, Shi L. Long non-coding RNA LUCAT1 modulates methotrexate resistance in osteosarcoma via miR-200c/ABCB1 axis. Biochem Bioph Res Co. 2018; 495(1): 947-953.

85. Kun-Peng Z, Xiao-Long $M$, Chun-Lin Z. LncRNA FENDRR sensitizes doxorubicin-resistance of osteosarcoma cells through down-regulating ABCB1 and ABCC1. Oncotarget. 2017; 8(42): 71881-71893.

86. Zhang $\mathrm{C}$, Zhu $\mathrm{K}$, Shen $\mathrm{G}$, et al. A long non-coding RNA contributes to doxorubicin resistance of osteosarcoma. Tumor Biology. 2016; 37(2): 2737-2748.

87. Zhu KP, Zhang CL. Sensitivity of doxorubicin-resistant osteosarcoma cells to doxorubicin regulated by long non-coding RNA NR_036444. Zhonghua Zhong Liu Za Zhi. 2017; 39(4): 250-255.

88. Zhang $\mathrm{C}$, Zhu K, Ma X. Antisense lncRNA FOXC2-AS1 promotes doxorubicin resistance in osteosarcoma by increasing the expression of FOXC2. Cancer Lett. 2017; 396(Jun): 66-75.

89. Wang Y, Zhang L, Zheng X, et al. Long non-coding RNA LINC00161 sensitises osteosarcoma cells to cisplatin-induced apoptosis by regulating the miR-645-IFIT2 axis. Cancer Lett. 2016; 382(2): 137-146.

90. Ma B, Yuan Z, Zhang L, et al. Long non-coding RNA AC023115.3 suppresses chemoresistance of glioblastoma by reducing autophagy. Biochimica et Biophysica Acta (BBA) - Molecular Cell Research. 2017; 1864(8): 1393-1404.

91. Zhang C, Ma Q, Shi $Y$, et al. A novel 5-fluorouracil-resistant human esophageal squamous cell carcinoma cell line Eca-109/5-FU with significant drug resistance-related characteristics. Oncol Rep. 2017; 37(5): 2942-2954.

92. Wen Q, Liu Y, Lyu H, et al. Long Noncoding RNA GAS5, Which Acts as a Tumor Suppressor via microRNA 21, Regulates Cisplatin Resistance Expression in Cervical Cancer. Int J Gynecol Cancer. 2017; 27(6): 1096-1108.

93. Zhang D, Ding L, Li Y, et al. Midkine derived from cancer-associated fibroblasts promotes cisplatin-resistance via up-regulation of the expression of IncRNA ANRIL in tumour cells. Sci Rep-Uk. 2017; 7(1): 16231.

94. Tan D, Chong FT, Leong HS, et al. Long noncoding RNA EGFR-AS1 mediates epidermal growth factor receptor addiction and modulates treatment response in squamous cell carcinoma. Nat Med. 2017; 23(10): 1167-1175.

95. Li W, Li H, Zhang L, et al. Long non-coding RNA LINC00672 contributes to p53 protein-mediated gene suppression and promotes endometrial cancer chemosensitivity. J Biol Chem. 2017; 292(14): 5801-5813.

96. Yan H, Xia JY, Feng FZ. Long non-coding RNA ENST00000457645 reverses cisplatin resistance in CP70 ovarian cancer cells. Genetics and Molecular Research. 2017; 16(1): 5801-5813.

97. Teschendorff AE, Lee $\mathrm{SH}$, Jones A, et al. HOTAIR and its surrogate DNA methylation signature indicate carboplatin resistance in ovarian cancer. Genome Med. 2015; 7: 108.

98. Yan H, Xia JY, Feng FZ. Long non-coding RNA ENST00000457645 reverses cisplatin resistance in CP70 ovarian cancer cells. Genetics and Molecular Research. 2017; 16(1).

99. Ren S, Li G, Liu C, et al. Next generation deep sequencing identified a novel lncRNA n375709 associated with paclitaxel resistance in nasopharyngeal carcinoma. Oncol Rep. 2016; 36(4): 1861-1867.

100. Zhou X, Wang W, Zhu W, et al. High expression of long non-coding RNAAFAP1-AS1 predicts chemoradioresistance and poor prognosis in patients with esophageal squamous cell carcinoma treated with definitive chemoradiotherapy. Mol Carcinogen. 2016; 55(12): 2095-2105.

101. $\mathrm{Xu} \mathrm{Z}$, Yang F, Wei D, et al. Long noncoding RNA-SRLR elicits intrinsic sorafenib resistance via evoking IL-6/STAT3 axis in renal cell carcinoma. Oncogene. 2017; 36(14): 1965-1977. 